\title{
Prosthetic Breast Reconstruction with Acellular Dermal Matrix
}

\author{
Katie Weichman and Joseph Disa \\ Additional information is available at the end of the chapter \\ http://dx.doi.org/10.5772/56331
}

\section{Introduction}

The use of prosthetic devices for breast reconstruction began in the early 1960's with siliconegel filled implants. Currently, traditional two-stage tissue expander to implant prosthetic breast reconstruction remains the most common type of breast reconstruction performed in the United States. Most recent ASPS statistics estimate greater than $70 \%$ of reconstructions are implant based.[1]

Over the years, implant technology and surgical techniques have evolved, resulting in the improved outcomes in breast reconstruction. National trends have moved away from total submuscular coverage toward "dual-plane" positioning of implants. Dual-plane placement provides multiple advantages including decreased chest wall morbidity and increased patient comfort. However, limitations of dual-plane positioning include lack secure coverage of the inferior pole of the implant, less control over the position of the inframammary fold (IMF), and a tendency towards superior migration of the pectoralis major muscle and expander during expansion. [2]-[4] Additional limitations of traditional tissue expander reconstruction remains the time required to reach maximal expansion and difficulty in inferior pole expansion. [5]

Acellular dermal matrix (ADM), was initially employed in breast surgery for revision breast augmentation to prevent rippling and contour abnormalities. It is currently being utilized to address the limitations associated with the dual plane and total submuscular techniques.[6] Its use in immediate implant based breast reconstruction became popular in 2005 after Brueing et al. published a case series describing its use as a sling to cover the inferior-lateral pole in immediate permanent implant reconstruction.[5],[4]Subsequently several case series were published to further support this technique and expand its use to two-stage tissue expander reconstruction. [3],[7]-[11]Proponents of this technique advocate two main advantages: inferior-lateral pole coverage of the implant where the pectoralis muscle is absent and greater 
initial tissue expansion. [7],[11]-[14][16]-[18]Other proposed advantages include decreased postoperative pain, decreased donor site morbidity, and improved aesthetic outcomes. Several disadvantages have been proposed in the literature including increased postoperative infectious complications, seroma, and explantation.[13],[19]-[21] (Figure 1)

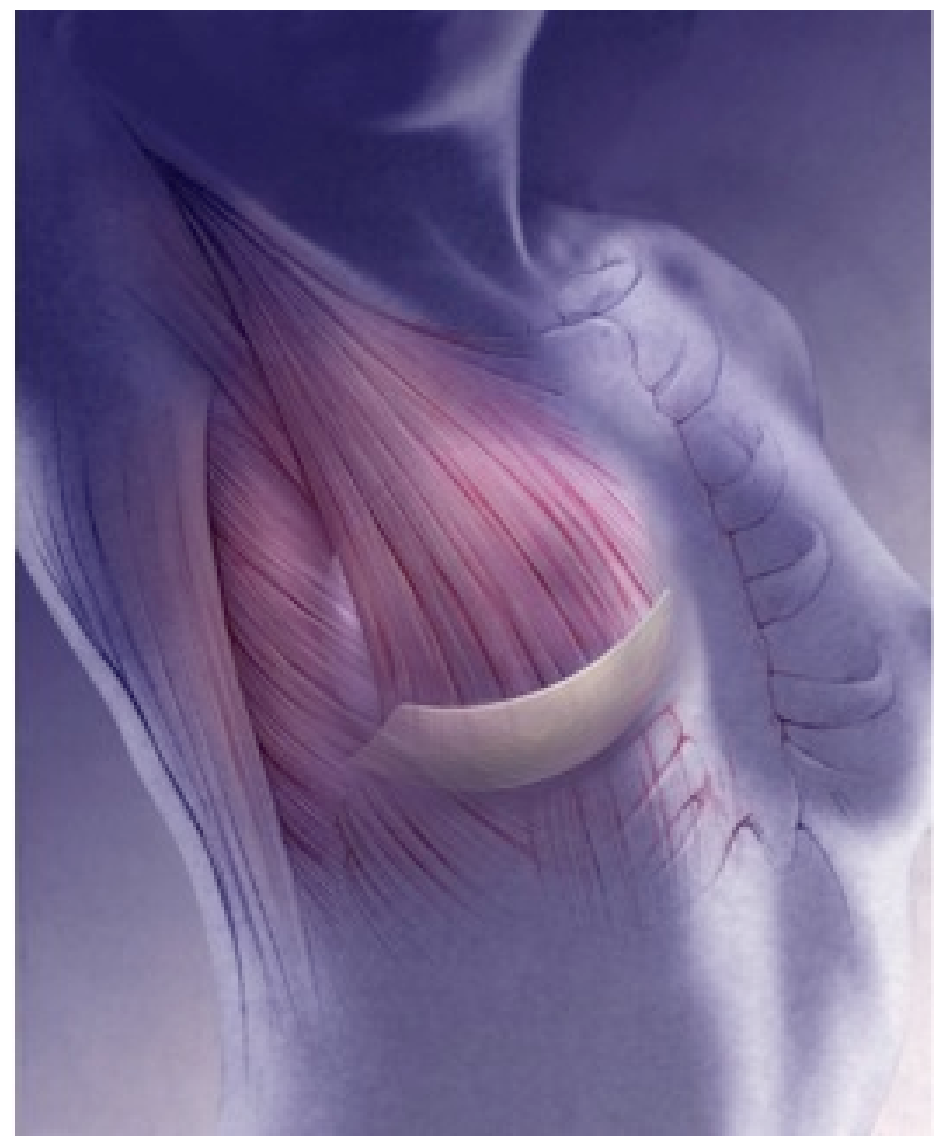

Figure 1. Placement of Accellular Dermal Matrix 


\section{Types of acellular dermal matrix}

While the majority of breast reconstruction with acellular dermal matrix has been described with the use of human matricies, porcine and bovine products are available and have been described for use. Human acellular dermal matricies published in the literature for use in breast reconstruction include; Alloderm (LifeCell, Branchburg, NJ), Flex HD (Ethicon, Sommerville, NJ), Neoform (Mentor, Santa Barbara, CA), DermaMatrix (Synthes, West Chester, PA). Porcine derived matrices include; Strattice (LifeCell, Branchburg, NJ) and Permacol (Covidien, Boulder, $\mathrm{CO}$ ). Currently there is only one bovine dermal matrix on the market, Surgimend (TEI Biosciences, Boston, MA).

\section{Timing}

Prosthetic breast reconstruction with acellular dermal matrix can be accomplished either in the immediate or delayed fashion. The advantage of immediate reconstruction is that the first step of breast reconstruction is accomplished at the time of the mastectomy under the same general anesthesia. In this setting, maximum amount so breast skin can be preserved as the prosthetic device will occupy some of the mastectomy space. In the setting of single stage breast reconstruction using a permanent implant, immediate reconstruction allows for the placement of an optimally sized device.

Delayed breast reconstruction using this technique is also possible, however, significantly more tissue expansion is generally necessary. In this method, the mastectomy skin flaps are re-elevated and expanded postoperatively to re-create a pocket for the ultimate placement of a permanent breast implant. Although delayed breast reconstruction with a tissue expander requires an intraoperative procedure, it benefits from simplification of the initial phase of patient management. In the setting of high-risk disease and patients who may require chemotherapy and radiation therapy, delayed reconstruction will not result in a delay of initiation of the adjuvant treatments.

\section{Patient selection}

While the majority of patients are candidates for prosthetic breast reconstruction and additionally prosthetic reconstruction with acellular dermal matrix. There are several limitations with the overall shape of the permanent implants that dictate the quality of the final result. Factors to consider include need for bilateral or unilateral reconstruction, the patients overall body habitus including body mass index (BMI) and chest width, comorbidities, and patients psychological profile. The ideal candidates for prosthetic reconstruction with acellular dermal matrix are thin patients undergoing bilateral breast reconstruction with adequate mastectomy skin flaps and thin patients with a non-ptotic breast undergoing unilateral reconstruction with 
adequate mastectomy skin flaps. In these situations, achieving reasonable symmetry is typically achievable.

However, as both breast size and degree of ptosis increases, symmetry in unilateral prosthetic breast reconstruction becomes more difficult to achieve. In this setting, patients may be candidates for contralateral symmetry procedures such as mastopexy or reduction mammoplasty. While the ultimate goal is to provide exact symmetry, patients should be aware that symmetry may only be accomplished in brassiere and clothing.

While there are no defined absolute contraindications for use of Acellular dermal matrix in prosthetic breast reconstruction, obesity, smoking history, and breast size $>600$ grams have been shown to be associated with increased rates of postoperative complications. [19]-[21]

\section{Technique}

The primary goal of prosthetic breast reconstruction is to achieve a breast mound that is symmetric with either the normal contralateral breast or the contralateral reconstruction. Clear communication between the ablative and reconstructive surgeons is necessary to achieve superior results. Mastectomy incisions are planned and marked together and the inframammary fold should additionally be marked and preserved when possible. Additionally, mastectomy skin flaps should be of adequate thickness to maintain blood supply to the skin and prevent mastectomy skin flap loss.

After mastectomy, careful hemostasis should be obtained within the mastectomy pockets. Then the inferolateral origin of the pectoralis major muscle, along with the investing fascia, is elevated off the anterior chest wall. Using electrocautery dissection, a subpectoral pocket is developed to the extent of the previously marked perimeter of the breasts. After the pocket has been successfully developed, an appropriately sized, usually $4-8 \mathrm{~cm} \times 14-16 \mathrm{~cm}$, sheet of acellular dermal matrix is prepared according to the manufacturer's recommendations. The $\mathrm{ADM}$ is then sutured to chest wall to recreated the inferior and lateral mammary folds. Most surgeons prefer the use of an absorbable suture including either 2-0 polydioxanone (PDS) (Ethicon, Somerville, NJ) or 2-0 Vicryl (Ethicon, Sommerville, NJ) suture. The inframammary $\mathrm{ADM}$ is curved laterally and cephalad along the lateral border of the breast perimeter to recreate the natural curvilinear origins of the inferolateral aspect of the detached pectoralis muscle and breast mound unit. Once the ADM has been secured to the inframammary fold, the width of the pocket is measured and tissue expander size is chosen based on the base width. Hemostasis in the pocket is then meticulously achieved. The tissue expander is then prepared in the standard sterile fashion on the back table and then placed in the pocket. The superior border of the ADM is sutured to the inferior and lateral border of the pectoralis muscle thus creating and inferolateral sling of ADM. (Figure 2 and Figure 3)

This tissue expander can be inflated intraoperatively to a volume determined appropriate by surgeon judgment. Care is taken to obliterate dead space but to not impart excessive pressure on the mastectomy skin flaps. One or two closed suction drains are utilized to drain the 


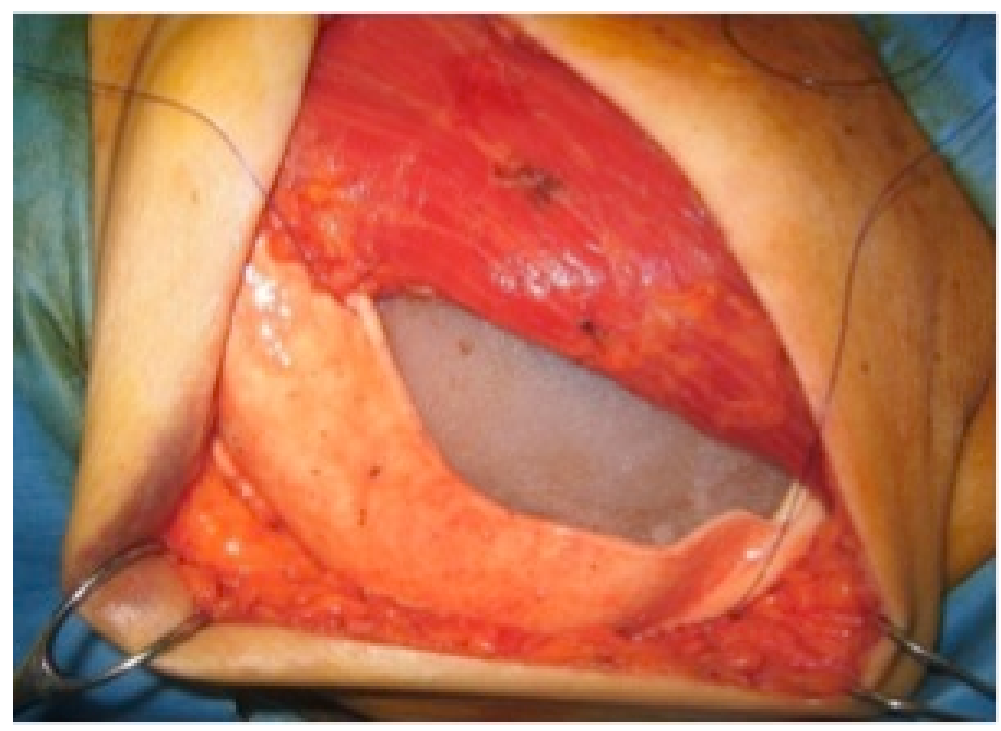

Figure 2. Intraoperative illustration of tissue expander placement into the pectoralis major and acellular dermal matrix pocket

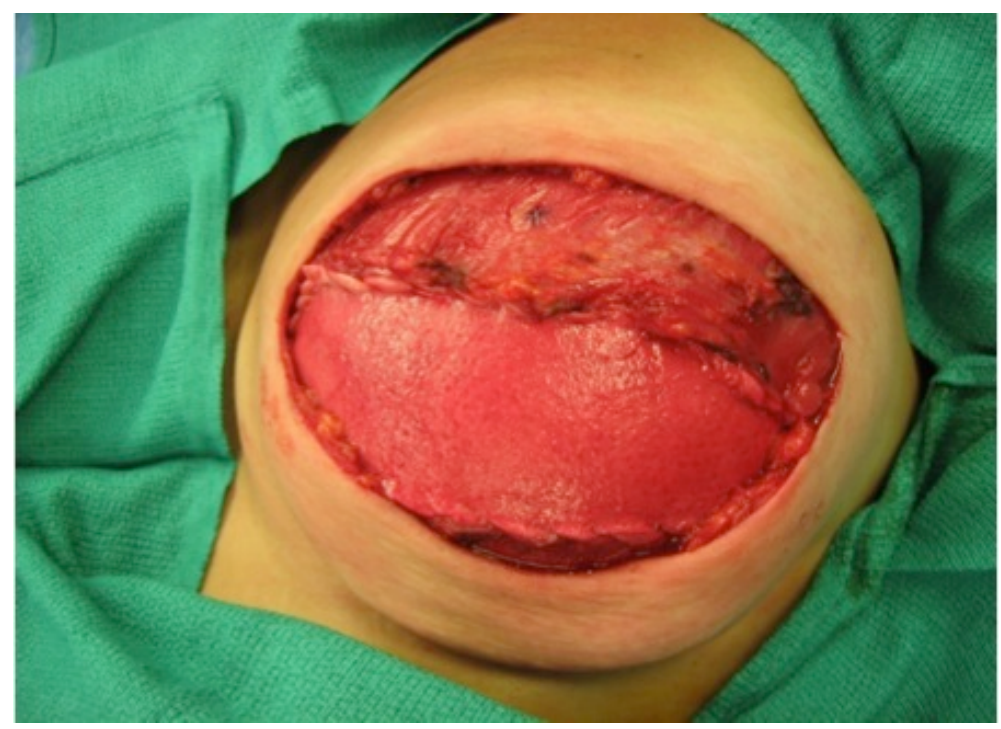

Figure 3. Intraoperative closure of pectoralis major and acellular dermal matrix over the tissue expander mastectomy space. The mastectomy skin flaps can be tailored to remove excess or non-viable

skin prior to final closure. (Figure 4) 


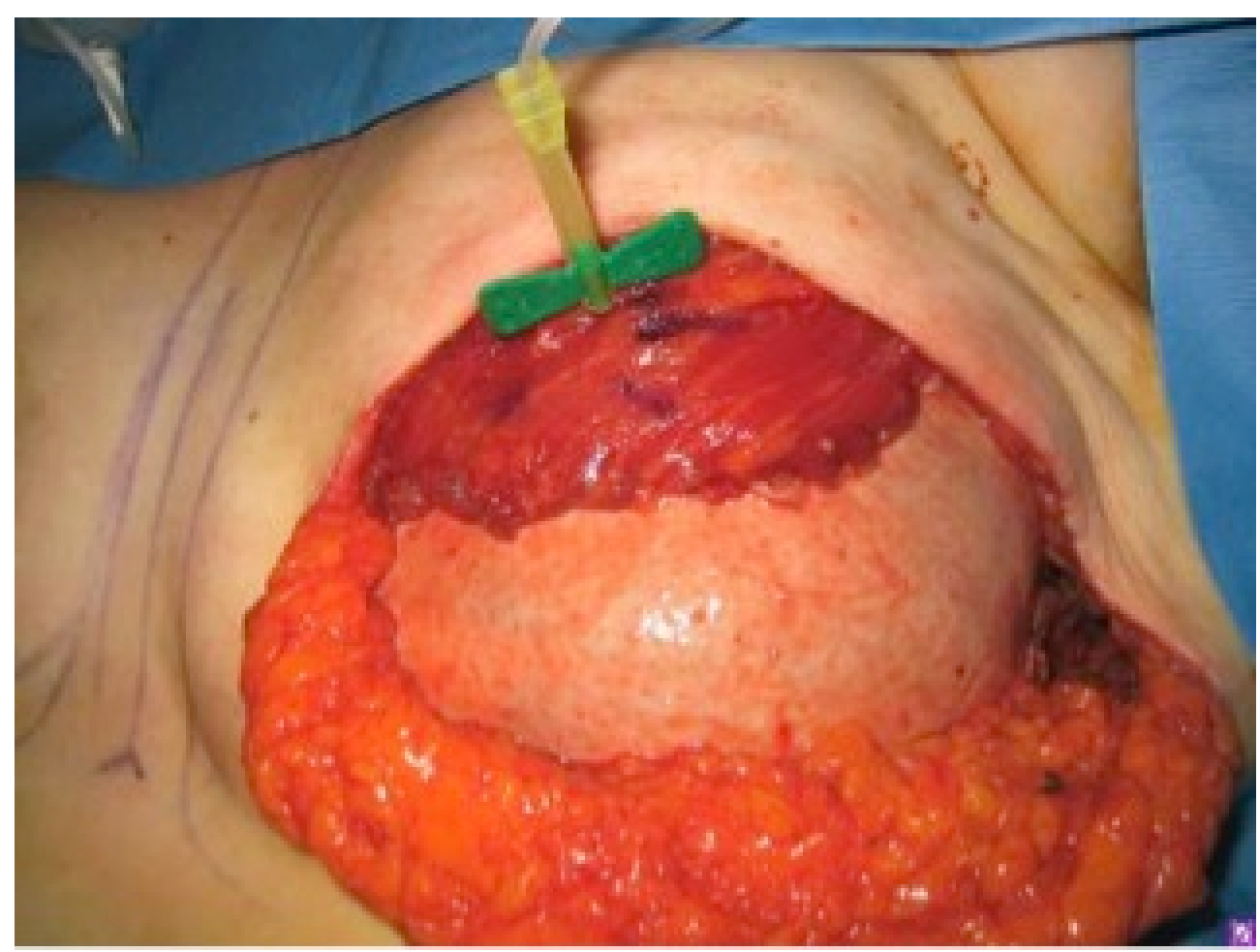

Figure 4. Intraoperative tissue expansion

\section{Expansion}

Tissue expansion begins in the office approximately 10-14 days after surgery when wound healing is stable and patient no longer has pain. Tissue expansion ensues in the standard fashion using the magnetic expander port finding device to find the site of the valve. The area is then cleansed with antiseptic solution and butterfly needle is used to gain access to the tissue expander. Approximately $30-120 \mathrm{~mL}$ of saline is injected into the expander during each expansion session. Expansions typically occur at weekly to monthly intervals. The final goal of expansion is guided by desired reconstruction breast size and/or achieving maximum symmetry with contralateral breast. Overexpansion (greater than tissue expander volume) helps to create ptosis in the secondary exchange procedure. In general, soft tissues are allowed to rest for at least one month between the time of last expansion and the implant exchange procedure. [22] (Figure 5) 


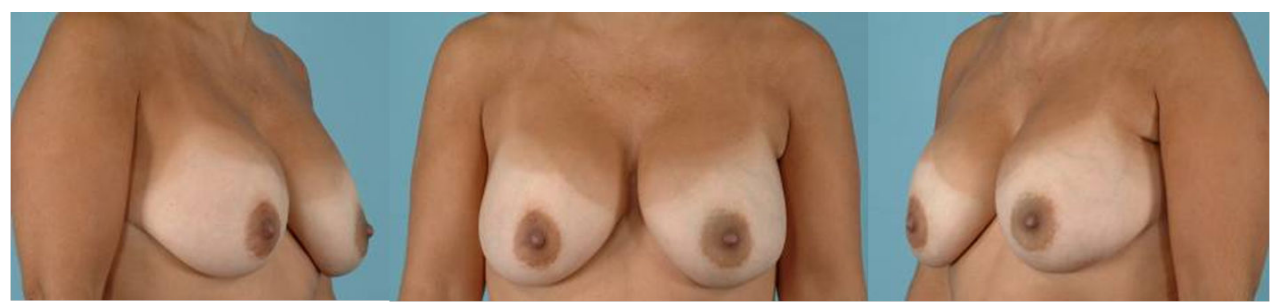

Before mastectomy

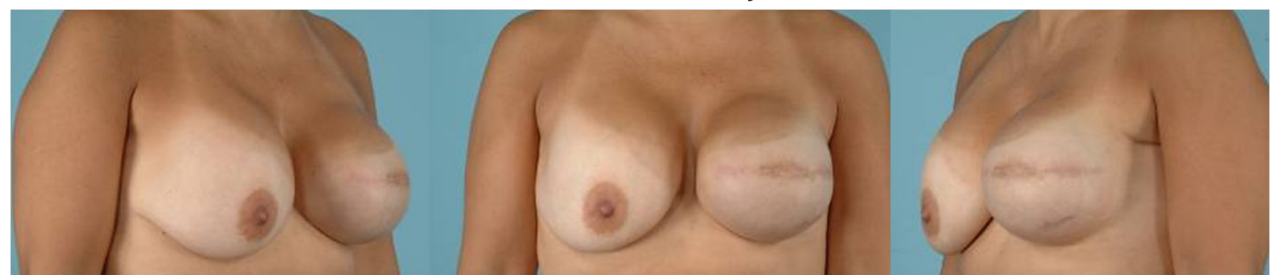

Post-TE

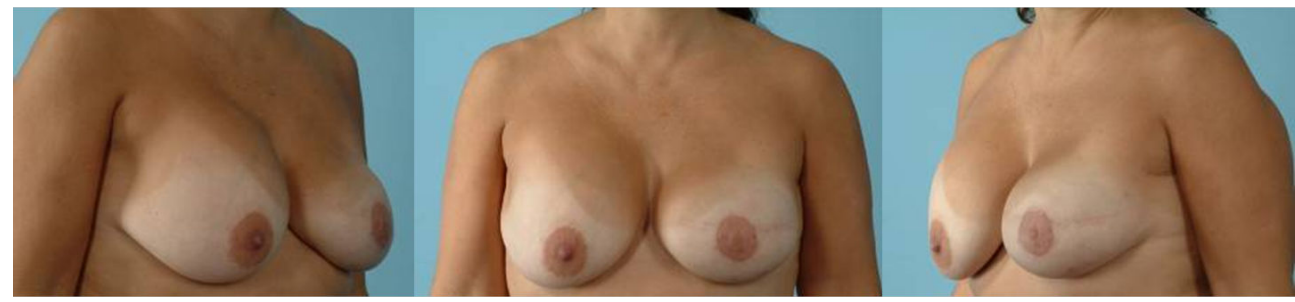

Post-implant

Figure 5. 49 year old female with left breast cancer who underwent left mastectomy with acellular dermal matrix and tissue expander followed by exchange for permanent implant.

\section{Proposed advantages}

ADM was initially described for use in single stage immediate permanent implant reconstruction and one proposed advantage of the use of ADM is to decrease or eliminate the use of tissue expanders. In the initial report by Breuing, and five subsequent retrospective studies, the efficacy and success of one stage reconstruction has been shown. [7],[9],[12],[14],[15],[23] In these retrospective reviews the overall complication rate is found to be between $6.9 \%$ and $25 \%$. [24] Breuing reports a $6.9 \%(2 / 30)$ complication rate in single state reconstructions and Zienowicz's et al. shows a complication rate of $25 \%$ (6/24) all of which were mastectomy skin flap necrosis, treated only with local wound care.[12],[25] The largest review of immediate one stage implant reconstructions by Colwell et al. describes an overall complication rate of $14.8 \%$ (49/331), including 9.1\% (30/331) cases of mastectomy skin flap necrosis. Of the cases of mastectomy skin flap necrosis five implants $(1.5 \%)$ required explantation. ${ }^{9}$ These results demonstrate the successful use of ADM as an adjunct to immediate one stage permanent implant reconstruction. However, it is important to realize, one stage reconstruction is not 
always possible in patients undergoing prosthetic reconstruction. In order to achieve maximum success in direct to implant reconstruction, proper patient selection is paramount. Specifically, the heath and viability of mastectomy skin flaps needs to be excellent and although it may be possible to make the reconstructed breast slightly larger than the native breast, ideal direct to implant candidates desire a similar or smaller sized reconstructed breast.[9]

Another proposed advantage of ADM is decreased postoperative pain associated with traditional dual plane or total submuscular implant placement due to less extensive muscle elevation and dissection.[5],[26] Several retrospective reviews in the literature have addressed this issue and while subjectively pain was shown to be diminished in patients being reconstructed with ADM. There are no studies that demonstrate objective data with statistical significance to support this claim. [10],[11],[15] However, a recent prospective randomized trial revealed no difference in postoperative pain when comparing total submuscular coverage to the patients undergoing reconstruction with ADM sling. [27]

Decreased operative time is another heralded advantage of ADM however, there is an absence of data in the literature proving this statement. There is anecdotal evidence in two case series suggesting this benefit. [5],[14]

Increased initial TE fill is a seemingly logical benefit to the use of acellular dermal matrix. The sling allows greater size of implant pocket and easier expansion within the pocket with less muscular recoil. This has been proven in many retrospective investigations with differences in initial tissue expander volumes as high as 300mL. [8],[12],[15]-[17],[19]-[21],[24] Two studies thwart this evidence, Preminger et al. comment that there is no statistically significant difference in expansion of the ADM cohort when compared to the non-ADM cohort at $224 \mathrm{~mL}$ versus $201 \mathrm{~mL}(\mathrm{p}=0.180)$. [28] However, this approaches significance and sample size alone may prove the limitation of this particular value. Additionally, Vardanian et al. saw a similar fill in the ADM cohort at $150 \pm 76 \mathrm{~mL}$ as compared to $100 \pm 69 \mathrm{~mL}$ in the non-ADM cohort.[29] However, it is important to realize that the factors contributing to increased tissue expander fill including body habitus, mastectomy skin flap condition, type of mastectomy performed (skin sparring, nipple areolar sparing), and surgeon judgment play roles in determining amount of tissue expander fill.

In addition to initial greater tissue expander fill, fewer number of overall expansions is another seemingly logical extension of the use of the ADM sling. Several studies have addressed this issue, however, the data is not as convincing. Four studies show that a statistically significant fewer numbers of expansions is required with the addition of ADM. [3,7,8,30] Sbitany shows a decreased total number of fills in the ADM group at 1.7 when compared to the non-ADM group at 4.3. ${ }^{8}$ Similarly, Nahabedian reports a mean number of expansions in the ADM group at 3 as compared to the non-ADM group at 5.5. [7] On the other hand four studies have shown no difference in the number of expansions when comparing each cohort. [10],[17],[27],[28] Seth demonstrates an average number of expansions in the ADM group of $4.8 \pm 2.4$ as compared to the non-ADM group of $5.3 \pm 2.4$ ( $\mathrm{p}=0.02$ ). [17] Similarly, McCarthy in a prospective randomized trial shows no difference in time to completion of expansion at 5.6 months in the ADM cohort when compared 4.6 months in the non-ADM cohort ( $p=0.93)$. [27] 
Several theories can explain the striking schism in the data regarding time to expansion, major factors accounting for these equivocal findings include surgeon technique and patients physical limitations. Some surgeons and institutions are more aggressive with tissue expansion volumes and time between expansions. Additionally, patients' ability to tolerate expansions is extremely variable. Given, this information, this proposed advantage is not likely clinically relevant.

Acellular dermal matrix was initially described for use in revision breast augmentation to treat capsular contractures. A natural translation would be to prevent capsular contractures in those undergoing breast reconstruction. Capsular contracture has been reported in rates as high as $14.1 \%$ in patients undergoing reconstruction without ADM.[31] Several authors have touted an absence of capsular contracture with ADM with follow up times ranging from 6.5 to 52 months. [5],[6],[11],[15],[23],[32] Vardanian et al. showed in a recent retrospective review that capsular contracture severity, as graded by modified Baker capsular contracture grading system, was significantly lessened in patient undergoing reconstruction with the addition of ADM.[29] Additionally, studies have not shown Baker capsular contracture grades greater than grade I or II.[10],[12] In a primate model Stump et al. evaluated the effect of ADM on capsule formation and found at 10 weeks there was no definable capsule in around the implants reconstructed with ADM compared to the implants without which had a definable capsule. To date, there is no long term data to support a protective effect of ADM to in reducing the incidence of capsular contracture.

Improved aesthetic outcomes of reconstructed breasts are the ultimate goal of plastic surgeons. However, evaluation of this outcome is very subjective and surgeon dependent. Many authors have argued that ADM provides better aesthetic outcomes but there is sparse data to support these claims. Two studies have objectively evaluated the aesthetic outcomes of breast reconstruction with ADM. Spear et al. defined a five-point scale and compared ADM reconstructed breast to contralateral non-reconstructed breasts. They found that scores for breast reconstructed with ADM did not significantly differ from the contralateral unreconstructed breast at 3.68 versus 3.98 ( $p=0.3$ ). [3] Additionally, Vardanian et al. showed that overall aesthetic outcomes, graded by four independent observers on a scale of 1-4, was statistically significantly greater in the ADM group at 3.26 when compared to the non-ADM group at 2.87. Additionally, the inframammary fold was found to be in better position in the ADM group at 3.35 as compared to the non-ADM group at 2.94. [29]

\section{Complications}

Complications associated with prosthetic breast reconstruction with acellular dermal matrix are similar to those reconstructed without acellular dermal matrix and should be divided into early and late complications. Early complications include; hematoma, seroma, mastectomy skin flap necrosis, infection, and need for explantation. Late complications include: asymmetry, implant wrinkling, malposition, capsular contracture, and late infection. 
The incidence of hematoma is generally accepted as less than $5 \%$. The treatment and consequences are similar with all breast reconstruction. This involves identification of hematoma and rapid evacuation to prevent sequelae, which includes mastectomy skin flap compromise, infection, and capsular contracture.

Seroma, unlike hematoma, is fraught with much controversy in the face of acellular dermal matrix. ADM is hypothesized to increase the risk of seroma and two studies show statistically significantly higher incidences of seroma. [13],[16] Chun shows seromas at an incidence of $14.1 \%$ in the ADM cohort when compared to non-ADM cohort at 2.7\%. [13] Similarly, Parks shows an incidence of $29.9 \%$ in the ADM cohort when compared to $15.7 \%$ in the non-ADM cohort.[16] However, there are many studies that do not show a statistically significant difference in the incidence of seroma associated with ADM. [17],[19]-[21],[29],[33] Many of these studies including Liu at 7.1\% in the ADM group versus $3.9 \%$ in the non-ADM group and Lanier at $13.4 \%$ in the ADM group versus $6.7 \%$ in the non-ADM group approach but fail to reach statistical significance. [19],[21] Given these findings it is important to take minimize the risk of seroma, including wide drainage with closed suction drains placed in the subcutaneous pocket plus or minus the expander/implant pocket and careful measurements of outputs to avoid premature drain removal.

Mastectomy skin flap necrosis is always a major concern in the setting of prosthetic breast reconstruction. Given the primary surgical intent of mastectomy is to treat breast cancer or remove all breast tissue to prevent cancer development, mastectomy skin flap thickness is often indeterminate. Additionally, other factors such as length of the mastectomy skin flap, medical comorbidities, smoking history, and surgical technique can often contribute to the development of mastectomy skin flap necrosis. Superficial or partial-thickness flap necrosis can be managed conservatively with local wound care. However, full thickness necrosis in the setting of an ADM assisted reconstruction should be managed with early excision with temporary deflation of expander to facilitated tension free closure and subsequent expansion.[34] Mastectomy skin flap necrosis associated with overt prosthetic exposure typically results in explantation. It is important to realize that ADM represents a second foreign body in addition to the expander/implant. Secondary infection of both the expander/implant and ADM is possible with the presence of mastectomy skin flap necrosis.

While there is no direct association between mastectomy skin flap necrosis and acellular dermal matrix, the incidence of mastectomy skin flap necrosis has been seen to be higher in patients undergoing reconstruction with ADM in two series. Weichman et al. reports a mastectomy skin flap necrosis rate of $8.3 \%$ in the ADM cohort as compared to $3.2 \%$ in the non$\mathrm{ADM}$ cohort and Chun et al. similarly shows an incidence of $23.4 \%$ in the ADM cohort as compared to $8.9 \%$ in the non-ADM cohort. [13],[20] Both series also show greater initial tissue expansion volume, which is thought to contribute to this increased incidence. Therefore, it is important to both prevent mastectomy skin flap necrosis through surgical judgment and careful inflation of initial tissue expander. Additionally, it is paramount to identify full thickness necrosis and treat expeditiously with excision and closure to prevent further sequlae. Some authors support the use of indocyanine green angiography to assess the mastectomy 
skin flap viability. However, the data to support this technology available in the literature is inconclusive.

Infectious complications of prosthetic breast reconstructions are cited as high as 35.4\%.[35],[36] Reconstruction with the addition of ADM poses a second foreign body and therefore infectious complications could be more likely. The literature displays divergent evidence with regards to infectious complications in the presence of ADM. There are a multitude of reports showing equivalent infectious complications when comparing ADM to non-ADM breast reconstructions. [3],[7-9],[15]-[18],[29],[37],[38]Conversely there are many reports showing increased infectious complications in patients with ADM reconstruction. [13],[19]-[21],[33] It is important to recognize infectious complications and treat expeditiously with either oral or intravenous antibiotics as per surgeon judgment.

\section{Conclusions}

Acellular dermal matrix is an influential addition to the plastic surgical armamentarium for breast reconstruction. Superior aesthetic results have been seen in patients undergoing both immediate one-stage implant reconstructions and two-stage tissue expander reconstruction. Surgeons should realize, however, that ADM is another tool in prosthetic breast reconstruction and not necessarily a panacea. Surgeon judgment based upon experience and evidence based best practices should guide the use of ADM in prosthetic breast reconstruction.

\section{Author details}

Katie Weichman ${ }^{1}$ and Joseph Disa ${ }^{2}$

1 New York University Medical Center, Institute of Reconstructive Plastic Surgery, New York, NY, USA

2 Memorial Sloan Kettering Cancer Center, New York, NY, USA

\section{References}

[1] Surgeons ASoP. American Society of Plastic Surgeons 2011 Statistics. 2011; http:// www.plasticsurgery.org/News-and-Resources/2011-Statistics-.html

[2] Spear SL, Majidian A. Immediate breast reconstruction in two stages using textured, integrated-valve tissue expanders and breast implants: a retrospective review of 171 consecutive breast reconstructions from 1989 to 1996. Plast Reconstr Surg. Jan 1998;101(1):53-63. 
[3] Spear SL, Parikh PM, Reisin E, Menon NG. Acellular dermis-assisted breast reconstruction. Aesthetic Plast Surg. May 2008;32(3):418-425.

[4] Spear SL, Pelletiere CV. Immediate breast reconstruction in two stages using textured, integrated-valve tissue expanders and breast implants. Plast Reconstr Surg. Jun 2004;113(7):2098-2103.

[5] Breuing $\mathrm{KH}$, Warren SM. Immediate bilateral breast reconstruction with implants and inferolateral AlloDerm slings. Ann Plast Surg. Sep 2005;55(3):232-239.

[6] Baxter RA. Intracapsular allogenic dermal grafts for breast implant-related problems. Plast Reconstr Surg. Nov 2003;112(6):1692-1696; discussion 1697-1698.

[7] Nahabedian MY. AlloDerm performance in the setting of prosthetic breast surgery, infection, and irradiation. Plast Reconstr Surg. Dec 2009;124(6):1743-1753.

[8] Sbitany H, Sandeen SN, Amalfi AN, Davenport MS, Langstein HN. Acellular dermisassisted prosthetic breast reconstruction versus complete submuscular coverage: a head-to-head comparison of outcomes. Plast Reconstr Surg. Dec 2009;124(6): 1735-1740.

[9] Colwell AS, Damjanovic B, Zahedi B, Medford-Davis L, Hertl C, Austen WG, Jr. Retrospective review of 331 consecutive immediate single-stage implant reconstructions with acellular dermal matrix: indications, complications, trends, and costs. Plast Reconstr Surg. Dec 2011;128(6):1170-1178.

[10] Namnoum JD. Expander/implant reconstruction with AlloDerm: recent experience. Plast Reconstr Surg. Aug 2009;124(2):387-394.

[11] Bindingnavele V, Gaon M, Ota KS, Kulber DA, Lee DJ. Use of acellular cadaveric dermis and tissue expansion in postmastectomy breast reconstruction. J Plast Reconstr Aesthet Surg. 2007;60(11):1214-1218.

[12] Zienowicz RJ, Karacaoglu E. Implant-based breast reconstruction with allograft. Plast Reconstr Surg. Aug 2007;120(2):373-381.

[13] Chun YS, Verma K, Rosen H, et al. Implant-based breast reconstruction using acellular dermal matrix and the risk of postoperative complications. Plast Reconstr Surg. Feb;125(2):429-436.

[14] Gamboa-Bobadilla GM. Implant breast reconstruction using acellular dermal matrix. Ann Plast Surg. Jan 2006;56(1):22-25.

[15] Salzberg CA. Nonexpansive immediate breast reconstruction using human acellular tissue matrix graft (AlloDerm). Ann Plast Surg. Jul 2006;57(1):1-5.

[16] Parks JR, Hammond SE, Walsh WW, Adams RL, Chandler RG, Luce EA. Human Acellular Dermis (ACD) vs. No-ACD in Tissue Expansion Breast Reconstruction. Plast Reconstr Surg. Jun 82012. 
[17] Seth AK, Hirsch EM, Fine NA, Kim JY. Utility of acellular dermis-assisted breast reconstruction in the setting of radiation: a comparative analysis. Plast Reconstr Surg. Oct 2012;130(4):750-758.

[18] Glasberg SB, Light D. AlloDerm and Strattice in breast reconstruction: a comparison and techniques for optimizing outcomes. Plast Reconstr Surg. Jun 2012;129(6): 1223-1233.

[19] Lanier ST, Wang ED, Chen JJ, et al. The effect of acellular dermal matrix use on complication rates in tissue expander/implant breast reconstruction. Ann Plast Surg. May; 64(5):674-678.

[20] Weichman KE, Wilson SC, Weinstein AL, et al. The use of acellular dermal matrix in immediate two-stage tissue expander breast reconstruction. Plast Reconstr Surg. May 2012;129(5):1049-1058.

[21] Liu AS, Kao HK, Reish RG, Hergrueter CA, May JW, Jr., Guo L. Postoperative complications in prosthesis-based breast reconstruction using acellular dermal matrix. Plast Reconstr Surg. May;127(5):1755-1762.

[22] Disa JJ, Ad-El DD, Cohen SM, Cordeiro PG, Hidalgo DA. The premature removal of tissue expanders in breast reconstruction. Plast Reconstr Surg. Nov 1999;104(6): 1662-1665.

[23] Breuing KH, Colwell AS. Inferolateral AlloDerm hammock for implant coverage in breast reconstruction. Ann Plast Surg. Sep 2007;59(3):250-255.

[24] JoAnna Nguyen T, Carey JN, Wong AK. Use of human acellular dermal matrix in implant- based breast reconstruction: evaluating the evidence. J Plast Reconstr Aesthet Surg. Dec 2011;64(12):1553-1561.

[25] Breuing KH, Colwell AS. Immediate breast tissue expander-implant reconstruction with inferolateral AlloDerm hammock and postoperative radiation: a preliminary report. Eplasty. 2009;9:e16.

[26] Topol BM, Dalton EF, Ponn T, Campbell CJ. Immediate single-stage breast reconstruction using implants and human acellular dermal tissue matrix with adjustment of the lower pole of the breast to reduce unwanted lift. Ann Plast Surg. Nov 2008;61(5):494-499.

[27] McCarthy CM, Lee CN, Halvorson EG, et al. The use of acellular dermal matrices in two-stage expander/implant reconstruction: a multicenter, blinded, randomized controlled trial. Plast Reconstr Surg. Nov 2012;130(5 Suppl 2):57S-66S.

[28] Preminger BA, McCarthy CM, Hu QY, Mehrara BJ, Disa JJ. The influence of AlloDerm on expander dynamics and complications in the setting of immediate tissue expander/implant reconstruction: a matched-cohort study. Ann Plast Surg. May 2008;60(5):510-513. 
[29] Vardanian AJ, Clayton JL, Roostaeian J, et al. Comparison of implant-based immediate breast reconstruction with and without acellular dermal matrix. Plast Reconstr Surg. Nov 2011;128(5):403e-410e.

[30] Parks JW, Hammond SE, Walsh WA, Adams RL, Chandler RG, Luce EA. Human Acellular Dermis versus No Acellular Dermis in Tissue Expansion Breast Reconstruction. Plast Reconstr Surg. Oct 2012;130(4):739-746.

[31] Spear SL, Newman MK, Bedford MS, Schwartz KA, Cohen M, Schwartz JS. A retrospective analysis of outcomes using three common methods for immediate breast reconstruction. Plast Reconstr Surg. Aug 2008;122(2):340-347.

[32] Becker S, Saint-Cyr M, Wong C, et al. AlloDerm versus DermaMatrix in immediate expander-based breast reconstruction: a preliminary comparison of complication profiles and material compliance. Plast Reconstr Surg. Jan 2009;123(1):1-6; discussion 107-108.

[33] Antony AK, McCarthy CM, Cordeiro PG, et al. Acellular human dermis implantation in 153 immediate two-stage tissue expander breast reconstructions: determining the incidence and significant predictors of complications. Plast Reconstr Surg. Jun;125(6): 1606-1614.

[34] Antony AK, Mehrara BM, McCarthy CM, et al. Salvage of tissue expander in the setting of mastectomy flap necrosis: a 13-year experience using timed excision with continued expansion. Plast Reconstr Surg. Aug 2009;124(2):356-363.

[35] Francis SH, Ruberg RL, Stevenson KB, et al. Independent risk factors for infection in tissue expander breast reconstruction. Plast Reconstr Surg. Dec 2009;124(6):1790-1796.

[36] Spear SL, Seruya M. Management of the infected or exposed breast prosthesis: a single surgeon's 15-year experience with 69 patients. Plast Reconstr Surg. Apr 2010;125(4):1074-1084.

[37] Ho G, Nguyen TJ, Shahabi A, Hwang BH, Chan LS, Wong AK. A systematic review and meta-analysis of complications associated with acellular dermal matrix-assisted breast reconstruction. Ann Plast Surg. Apr 2012;68(4):346-356.

[38] Kim JY, Davila AA, Persing S, et al. A meta-analysis of human acellular dermis and submuscular tissue expander breast reconstruction. Plast Reconstr Surg. Jan 2012;129(1):28-41. 